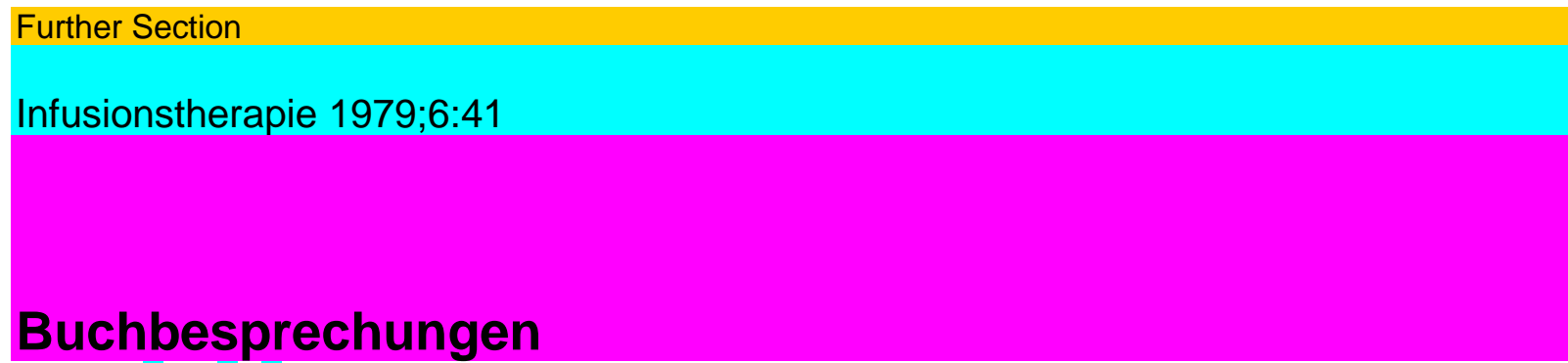

Praktische Chirurgie Heft 91 Nachoperationen an den Gallenwegen

W.Hess

Ferdinand Enke Verlag, Stuttgart 1977

X, 134 Seiten, 148 Abbildungen, kart. DM 58-

Zweiteingriffe an den Gallenwegen sind dann notwendig, wenn die Erstoperation nicht erfolgreich war. Sie stellen da-mit eine »Schattenseite« der modernen Chirurgie dar, wie der Autor des Buches selbst formuliert hat. Korrektureingriffe sind verantwortungsvoll und schwierig und sollten nur vom Er-fahrenen durchgeführt werden; sie sind keine Anfängeroperation. W. Hess, international anerkannter Spezialist auf dem Gebiet der Gallen- und Pankreaschirurgie, stellt dies ganz klar heraus.

Die »Nachoperationen an den Gallenwegen« gliedern sich in ein allgemeines Kapitel, in dem das sogenannte Post-Chole-zystektomie-Syndrom, diagnostische Verfahren, die Indika-tionsstellung und die allgemeine Operationsvorbereitung ab-gehandelt werden. Diesem folgen Abschnitte über Reopera-tionen nach unvollständiger Erstoperation (zurückgelassene Steine, unterlassene Papillotomie) und Nachoperationen bei Gallenfisteln. Breiten Raum nimmt das Kapitel »latrogene Schädigungen der Gallenwege« ein. Sie führen meist zu Ste-nosen der ableitenden Gallenwege mit schweren Folgen und bedürfen oft mehrfach operativer Korrekturen. Die Ursachen der iatrogenen Schäden und ihre operative Beseitigung werden im Detail beschrieben. Es ist sehr zu begrüßen, daß der Autor das hervorragende Operationsverfahren zur Gallenwegsrekonstruktion von Couinaud-Hepp besonders herausstellt. Es folgen Kapitel über Reoperationen nach biliodigestiven Anastomosen, nach Sphinkterotomie, nach vaskulären Schädigungen und nach Tumorrezidiven. Das Buch schließt mit einer Statistik der Ein-griffe, Todesfälle und Komplikationen. Grundlage bildet hierfür das große Erfahrungsmaterial von 310 Sekundärope-rationen.

Die vorliegende Schrift füllt eine Lücke in der chirurgischen Bibliographie und ist daher jedem auf dem Gebiet der Gallen-chirurgie tätigen Chirurgen wärmstens zu empfehlen. Hervorzuheben sind die klare Gliederung und die detaillierte Be-schreibung der Operationstechniken, die durch vorziigliche Strichzeichnungen (I. Schaumburg) veranschaulicht werden. Hier zeigt sich die große klinische und operative Erfahrung des Autors. Die verschiedenen Komplikationen und Folgen nach Gallenoperationen werden durch klinische Beispiele be-legt und - soweit notwendig - durch typische Röntgenbilder ergänzt.

Der Referent hat das Buch selbst mit großem Gewinn gele-sen. Für eine eventuelle Zweitauflage des Buches würde er sich noch ein zusätzliches Kapitel über Begutachtungsfragen wünschen. Eine weitere Anmerkung sei noch erlaubt: Man sollte die Choledochoduodenostomie wegen möglicher schwerwiegender Komplikationen grundsätzlich nicht mehr bei gutartigen Erkrankungen des Gallenwegsystems empfehlen (s. Seite 16), sondern ausschließlich als Palliativmaßnah-me für Tumorstenosen reservieren.

R. Häring, Berlin

Leitfaden der Infusionstherapie 


\section{H. GOFFERJE}

F. K. Schattauer Verlag, Stuttgart/New York 1978

X, 179 Seiten, 26 Abbildungen, 91 Tabellen, DM 17,80

Die moderne Infusionstherapie einschließlich der parentera-len Ernährung gehört heute zu den unentbehrlichen thera-peutischen Hilfsmitteln des klinisch tätigen Arztes. Durch ihren gezielten Einsatz können viele Krankheitsverläufe gün-stig beeinflußt werden. Trotzdem wird die Bedeutung der Infusionstherapie im Rahmen der medizinischen Ausbildung noch nicht entsprechend berücksichtigt. Nach neuesten Um-fragen setzen sich nur etwa $10 \%$ der klinisch tätigen Ärzte intensiver mit den Problemen der Infusionstherapie ausein-ander. Das vorliegende Buch beschränkt sich bewußt auf eine Vermittlung des unbedingt erforderlichen klinisch relevan-ten Wissens. Es ist deshalb besonders geeignet auch die Ärzte anzusprechen die sich bisher mit den Problemen der Infusionstherapie nur am Rande beschäftigt haben. Ausgehend von physiologischen und pathophysiologischen Zusammen-hängen werden Anleitungen für eine adäquate Infusionstherapie in verschiedenen klinisch wichtigen Situationen erar-beitet. Die wesentlichen diagnostischen und therapeutischen Fakten werden übersichtlich in Abbildungen und Tabellen zu-sammengefaßt. Dadurch wird dem Leser ein rascher Zugang zu dem komplexen Gebiet der Infusionstherapie und parente-ralen Ernährung eröffnet. Einmal Gelesenes kann rasch wie-der aufgefunden und aufgefrischt werden. Dazu trägt auch das ausführliche gut gegliederte Sachverzeichnis bei. Wer sich eingehender mit Einzelproblemen beschäftigen will

findet im Anschluß an jedes Kapitel entsprechende Literaturhin-weise. Das Buch ist alien Kollegen

die ohne großen Zeitauf-wand ein Basiswissen auf diesem Gebiet erwerben wollen zu empfehlen.

H. TIEFEL

Erlangen 